\title{
SERIES INTRODUCTION \\ The cellular response to aggregated proteins associated with human disease
}

\author{
David H. Perlmutter \\ University of Pittsburgh School of Medicine and Children's Hospital of Pittsburgh, Pittsburgh, Pennsylvania, USA \\ J. Clin. Invest. 110:1219-1220 (2002). doi:10.1172/JCI200216780.
}

Although there have been major conceptual advances in understanding mechanisms of protein folding and the role of chaperones since the work of Anfinsen 30 years ago (1), the past few years have witnessed an explosion of new information about protein folding and about the mechanisms by which disease-associated proteins aggregate, injure cells, and activate an elaborate signal transduction machinery designed to protect host cells from such injury. This Perspective series is intended to describe these advances and their impact on a number of important diseases, such as Alzheimer and several other neurodegenerative diseases, as well as cystic fibrosis (CF) and $\alpha 1$-antitrypsin ( $\alpha 1$-AT) deficiency.

In the first Perspective, Horwich reviews what is known about how chaperones facilitate protein folding. Recent studies have shown that protein aggregates are not random, nonspecific entities, but rather "specific, organized, polymeric structures formed from partly structured folding intermediates by alternative, offpathway, folding steps" (2). The concept that protein aggregates have a higher-order structure has extremely important implications for disease. Indeed, Bucciantini et al. have recently shown that when harmless proteins are induced to form amyloid-like fibrillar aggregates under specified conditions in vitro, it is the oligomeric structures formed early in the aggregation process, and not the final fibrillar structures, that are toxic to cells (3). In the second Perspective in this series, Selkoe (4) describes the potential importance of this concept for the mechanism of neurotoxicity in Alzheimer disease. Neuronal injury and degeneration in Alzheimer disease follow the appearance of aggregates of the amyloid- $\beta$ peptide and the microtubule-associated phosphoprotein tau in the brain. The amyloid- $\beta$ peptide is generated from the amyloid- $\beta$ protein precursor (APP) by a complex posttranslational processing pathway that

\footnotetext{
Address correspondence to: David H. Perlmutter, Department of Pediatrics, Children's Hospital of Pittsburgh, 3705 Fifth Avenue, Pittsburgh, Pennsylvania 15213-2583, USA. Phone: (412) 692-8071; Fax: (412) 692-5946 E-mail: perldav@chp.edu.

Conflict of interest: No conflict of interest has been declared. Nonstandard abbreviations used: $\alpha 1$-antitrypsin ( $\alpha 1-\mathrm{AT}$ ); endoplasmic reticulum (ER); unfolded protein response (UPR); cystic fibrosis (CF).
}

involves a number of proteolytic cleavage steps, including one that involves presenilins -1 and -2 . Mutations in APP and presenilins have been associated with earlyonset Alzheimer dementia. New studies from Selkoe's laboratory now show that dimers and trimers of amyloid- $\beta$ peptide are toxic to neurons and impair synaptic function but that the fully aggregated forms, protofibrils and fibrils, do not have these properties (5).

Much has been learned in recent years about how cells respond to and attempt to protect themselves from aggregated proteins that accumulate in the endoplasmic reticulum (ER). As part of its quality control function, the ER possesses machinery whereby it can recognize unassembled or aggregated proteins and degrade them - the ER-associated degradation (ERAD) pathway. Although this pathway was originally thought to involve a distinct proteolytic mechanism, it now appears to be mediated in large part from the cytoplasmic face of the ER by the ubiquitin system and the proteosome. Substrate-specific and cell type-specific nonproteosomal mechanisms also contribute to ER degradation. Several mechanisms for transport of aggregated proteins from the ER to the cytoplasm have been proposed in the literature, including retro-translocation through the import channel (reviewed in ref. 6) and proteosomemediated "extraction" or "dislocation" (7).

The ER also responds to the presence of aggregated proteins by activating the unfolded protein response (UPR). Articles in this series by Kaufman (8) and by Ron (9) describe quite remarkable discoveries about this response mechanism. The UPR is a signal transduction pathway in which the accumulation of unfolded proteins in the ER activates a change in expression of a repertoire of gene products that is designed to facilitate the capacity of the cell to adapt to increased load in the ER. Although it was originally believed that the UPR only resulted in upregulation of molecular chaperones in the ER, such as BiP and GRp94, recent studies have shown that many other factors that participate in protein folding, protein translocation, protein degradation, and generating structural components of the ER membrane are transcriptionally activated by the UPR. Furthermore, the UPR is associated with repression of translation as an adaptation to decrease the accumulation of unfolded proteins in the ER. Ron (9) reviews new 
information indicating that the UPR plays a role in determining ER size and function during cell- and tissue-specific differentiation and morphogenesis, new information about the role of the UPR in other forms of cellular stress such as ischemia, viral infection, and inherited diseases that alter protein folding, and new information about the role of the UPR in the survival of stressed cells. Indeed, one recent study of a mutant form of insulin in a mouse model of diabetes has provided evidence for the concept that the cellular response to "ER stress" is itself directly responsible for cell injury and death (10).

In most cases of $\mathrm{CF}$, the mutant chloride channel CFTR $\Delta$ F508 is not translocated to the apical membrane of epithelial cells, because it is defective in folding. CFTR $\Delta$ F508 is rapidly degraded in the ER by a mechanism that involves the ubiquitin-dependent proteosomal system. When the proteosome is pharmacologically inhibited, ubiquitinated CFTR $\Delta$ F508 aggregates in the cytoplasm to form structures that have been called aggresomes, but it is not clear whether this type of aggregation occurs in vivo or contributes to the pathobiology of CF. In the fifth Perspective in this series, Gelman and Kopito discuss the possibility that chemical chaperones or other pharmacological strategies can be used to rescue the folding defect underlying CF (11).

In $\alpha 1-A T$ deficiency, an aggregated mutant protein is retained in the ER of liver cells causing chronic hepatic injury and hepatocellular carcinoma in a subgroup of affected homozygotes. In the sixth Perspective in this series, Lomas and Mahadeva review progress in understanding how the mutant $\alpha 1$-ATZ molecule that characterizes this deficiency polymerizes into a higher-order structure (12). These revelations have been made possible by studies of the structure and function of $\alpha 1$-AT and other members of the serpin family, as well as studies of the mechanisms by which serpins inhibit proteinases and/or interact with other proteins (12). In fact, Lomas and colleagues have shown that polymerization of a mutant form of the neuron-specific neuroserpin results in its retention in the ER and severe dementia in familial encephalopathy with neuroserpin inclusion bodies (13). In the next Perspective, I describe recent work indicating that liver cells respond to the accumulation of mutant $\alpha 1-\mathrm{ATZ}$ in the ER by activating autophagy, the pathway by which cells digest senescent or damaged intracellular organelles and constituents (14). The autophagic response may be a key mechanism by which cells rid themselves of aggregated proteins. It has recently been implicated in the gain-of-function disease caused by mutant peripheral myelin protein- 22 in Schwann cells in Charcot-Marie-Tooth disease and Dejerine-Sottas syndrome (15). This Perspective also discusses evidence that retention of aggregated proteins in the ER, and that the ER stress response in general, leads to mitochondrial dysfunction, perhaps as the common final pathway for cellular injury and death.

In addition to providing further understanding of the pathobiology of diseases associated with aggregated proteins, the research described in this series has led to several novel concepts for chemoprophylaxis and therapy that could be translated into clinical care in the near future, including strategies for preventing protein aggregation and for enhancing disposal and/or clearance of the toxic protein aggregates, as well as for pharmacologically blocking mechanisms of cytotoxicity elicited during protein aggregation.

1. Anfinsen, C.B. 1973. Principles that govern the folding of protein chains. Science. 181:223-230.

2. Horwich, A. 2002. Protein aggregation in disease: a role of folding intermediates forming specific multimeric interactions. J. Clin. Invest. 110:1221-1232. doi:10.1172/JCI200216781.

3. Bucciantini, M., et al. 2002. Inherent toxicity of aggregates implies a common mechanism for protein misfolding diseases. Nature. 416:507-511.

4. Selkoe, D.J. 2002. Deciphering the genesis and fate of amyloid- $\beta$ protein yields novel therapies for Alzheimer disease. J. Clin. Invest. In press.

5. Walsh, D.M., et al. 2002. Naturally secreted oligomers of amyloid ? protein potently inhibit hippocampal long-term potentiation in vivo. Nature. 416:535-539.

6. Fewell, S.W., Travers, K.J., Weissman J.S., and Brodsky, J.L. 2001. The action of molecular chaperones in the early secretory pathway. Annu. Rev. Genet. 35:149-191.

7. Mayer, T., Braun, T., and Jentsch, S. 1998. Role of the proteosome in membrane extraction of a shortlived ER-transmembrane protein. EMBO J. 17:3251-3257.

8. Kaufman, R. 2002. Orchestrating the unfolded protein response in health and disease. J. Clin. Invest. In press.

9. Ron, D. 2002. Translational control in the endoplasmic reticulum stress response. J. Clin. Invest. In press.

10. Oyadomari, S., et al. 2002. Targeted disruption of the Chop gene delays endoplasmic reticulum stress-mediated diabetes. J. Clin. Invest. 109:525-532. doi:10.1172/JCI200214550.

11. Gelman, M.S., and Kopito, R.R. 2002. Rescuing protein conformation: prospects for pharmacological therapy in cystic fibrosis. J. Clin. Invest. In press.

12. Lomas, D.A., and Mahadeva, R. 2002. $\alpha 1$-Antitrypsin polymerization and the serpinopath: pathobiology and prospects for therapy. J. Clin. Invest. In press.

13. Davis, R.L., et al. 1999. Familial dementia caused by polymerization of mutant neuroserpin. Nature. 401:376-379.

14. Perlmutter, D.H. 2002. Liver injury in $\alpha 1$-antitrypsin deficiency: role of autophagy and mitochondrial injury in response to aggregated protein in the endoplasmic reticulum. J. Clin. Invest. In press.

15. Dickson, K.M., et al. 2002. Association of calnexin with mutant peripheral myelin protein-22 ex vivo: a basis for "gain-of-function" ER diseases. Proc. Natl. Acad. Sci. USA. 99:9852-9857. 